\title{
Production of Oriented Fibers out of Poly(Hydroxybutyrate/Hydroxyvalerate) Copolymers and Testing of Mechanical Stability under Static and Cyclic Loads
}

\author{
Tatiana G. Volova ${ }^{\mathrm{a}, \mathrm{b}, *}$, Sergei A. Gordeev \\ and Ekaterina I. Shishatskaya ${ }^{a}$ \\ ${ }^{a}$ Institute of Biophysics of Siberian Branch of Russian Academy of Sciences, \\ Akademgorodok, Krasnoyarsk, 660036 Russia \\ ${ }^{b}$ Siberian Federal University, \\ 79 Svobodny, Krasnoyarsk, 660041 Russia \\ ${ }^{c}$ Department of Cardiac Surgery University of Glasgow, \\ 10 Royal Infirmary, Alexandra Parade G3, UK ${ }^{1}$
}

Received 2.06.2008, received in revised form 25.08.2008, accepted 27.08.2008

We investigated the process of production of oriented fibers by melt spinning of biodegradable hydroxybutyrate and hydroxyvalerate (PHBV) copolymers. It was revealed that temperature has a large effect on the stability of extrusion and quality of fibers, and the optimal paramter values were found. We showed that the diameter of fibers and orientation degree depend on the spinning rate which can vary in a wide range (from 2-3 to $40 \mathrm{~m} / \mathrm{min}$ ), not affecting the quality of fibers. We studied the extensibility and deformability of fibers at different temperatures and found the conditions allowing to produce fibers with the diameter of 0.17-0.20 $\mathrm{mm}$ with high mechanical characteristics (strength 306 $M P a$, elasticity modulus $3 \mathrm{GPa}$, elongation at break 24\%), that were stable during the 3 month period of observation. We obtained and analyzed extension curves on which three areas were fixed: linear elasticity, visible deformation, totally nonreversible deformation, which is typical for the elongation curves of polymer elastomers. It was stated that the produced fibers have high mechanical stability at static and cyclic loading conditions (up to $100 \mathrm{MPa}$ ); in the area of linear elasticity the deformation of fibers is practically totally reversible after 1000 cycles of loading.

Keywords: Polyhydroxyalkanoates, Processing, Fiber, Production, Properties.

\section{Introduction}

Polyhydroxyalkanoates

biodegradable polyesters of microbiological origin, can be processed by a variety of generally accepted methods (gel-spun technology, solution casting, electrostatic extrusion, solution press molding etc.) (Amass, 1998; Volova, 2004). The range of temperatures at melting point $\left(150^{\circ} \mathrm{C}\right)$ and at the onset of decomposition $\left(270{ }^{\circ} \mathrm{C}\right)$ is an essential technological property of PHA, as it makes possible production of various items out of them (films, sutures, hollow forms) using generally accepted polymer processing thermal methods. At the same time it should be mentioned

\footnotetext{
Corresponding author E-mail address: volova45@mail.ru (C) Siberian Federal University. All rights reserved
} 
that PHA are rather difficult to process because of their relatively low decomposition temperatures near their melting points and brittleness. The low rate of PHA nucleation leads to formation of large spherulites inside of polymers which can deteriorate the physico-mechanical properties of the products. Various approaches such as use of plasticizers and modification of the polymer structure as well as preparation of blends and composites of different compounds are used to improve this situation (Asrar and Pierre, 2000; Ishikawa, 1996)

PHA are successfully used for production of oriented materials and products in the form of films and fibers. In one of the first works (Williams and Peoples, 1997) it was shown that PHA can be used for production of monofilaments which are resistant to long-time exposure in biological media; the fibers had the following strength characteristics: elasticity modulus $4.2 \mathrm{KPa}$; tensile strength 220-230 MPa. A lot of attention is given to the problem of optimization of fiber production process out of PHA. To improve the strength characteristics of polymer fibers, the process of production of monofilaments out of PHB melt was studied and the structure and properties of such fibers were investigated (Yamada and Kan, 1995; Yamamoto et al., 1997). Using X-Ray it was stated that polyhydroxybutyrate in these fibers is represented by two modifications of crystals and has bimodal chain orientation. Chain axes of the first population crystals are oriented mainly along the fiber axis, while chain axes of the second population crystals are oriented crosswise. During the hardening of the products at $100^{\circ} \mathrm{C}$ that followed, the population of the first type crystals dominates the second population, but increase of the temperature leads to a change in the ratio in favor of the second population. The strength of the fibers with an increased number of crosswiseoriented chains after hardening is up to $260 \mathrm{MPa}$. In a series of subsequent works it was shown that monofilaments produced by melt spinning or by PHB solution spinning can have satisfactory physico-mechanical properties - tensile strength up to 300-360 MPa and elasticity modulus up to $10 \mathrm{GPa}$, as well as a high degree of reversible deformation (up to 3 r/d) $[3,5,6,8]$. Recently the Japanese researchers leaded by Professor Doi managed to increase the mechanical strength of PHB monofilaments using a new original "colddrawn" technology (Iwata et al., 2002).

It is well known that, apart from adding nucleating agents, the presence of hydroxyvalerate in PHA, which reduces crystallinity and material crystallization time, contributes to a slower and more successful orientation of the polymer during processing and allows to make more stable oriented products (Katsuhiko, 1994).

The present work is aimed at production and investigation of fibers made out of hydroxybutyrate and hydroxyvalerate (PHBV) copolymer melts.

\section{Materials and Methods}

\section{Preparation of pure PHBV copolymer}

The tested material was the PHBV samples synthesized by the bacteria Ralstonia eutropha B5786. The strain is registered in the Russian Collection of Industrial Microorganisms. The method of bacteria cultivation is described in the work (Volova et al., 2007). PHBV is extractable from bacterial biomass due to their ability to dissolve in organic solvents and to be then precipitated by alcohols. The PHBV were extracted from bacterial biomass with chloroform and precipitated with ethanol. The extraction of PHBV from biomass was conducted in several stages. At the first stage, to partially destroy the cell wall and attain a fuller extraction of lipids, the bacterial biomass was centrifuged (for 15 $\mathrm{min}$ at $6000 \mathrm{rpm}$ ), collected, and covered with ethanol, $\mathrm{pH}$ 10.5-11.0 (0.5-0.7 g KOH/L ethanol). The sample was boiled using backflow condenser for $30 \mathrm{~min}$. Then the alcohol was removed, the 
biomass was covered with $86 \%$ ethanol and separated from alcohol by centrifuging. At the next stage the partly destroyed and defatted biomass was covered with chloroform and boiled for 30-40 min using a water bath with a backflow condenser. The sample was cooled and placed into a funnel to separate the chloroform extract of the polymer from the biomass. After separation of the phases, the polymer was precipitated by adding ethanol as a reagent. The procedure of redissolution and further precipitation of polymers was repeated several times to prepare specimens that would not contain organic impurities of protein, carbohydrate or lipid nature. The material mark was registered - Bioplastotan ${ }^{\mathrm{TM}}$ (Trademark № 315652, 2006). All the organic solvents used in the procedure were preliminarily distilled to remove impurities. Copolymer samples with different inclusions of hydroxyvalerate $(10,15$ and $21 \mathrm{~mol} \%$ ) were taken for investigation.

\section{Investigation of physical and chemical properties of PHAs}

The molecular mass of the polymer was measured in an Ubbellohde viscometer, with capillary diameter $0.34 \mathrm{~mm}$, at $30^{\circ} \mathrm{C}$. PHAV solutions in chloroform were used, with the polymer concentration ranging between 0.25 and $1.0 \mathrm{~g} \mathrm{l}^{-1}$. The degree of crystallinity was measured on a D8 ADANCE X-ray spectrometer (Bruker, Germany; graphite monochromator on a reflected beam) in a scan-step mode, with step $0.04^{\circ}$, exposure time $2 \mathrm{~min}$. The operating mode of the instrument was $40 \mathrm{kV} \times 40 \mathrm{MA}$.

\section{Preparation of fibers}

Monofilaments were produced using melt spinning with the help of single-screw extruder with round nozzle (diameter $1 \mathrm{~mm}$ ) made by Brabender Company (Germany). To optimize the process at the first stage, the following main parameters were used (temperature distribution by extruder zones, spinning rate, length and temperature of the receiving pit, reception speed). The physico-chemical characteristics of the fibers were studied with the help of Instron 1122 at room temperature; the initial length of samples - from 10 to $50 \mathrm{~mm}$; rate of extension $-100 \mathrm{~mm} / \mathrm{min}$.

\section{Results}

To produce fibers we used PHBV samples with different inclusions of hydroxyvalerate that had similar values of molecular mass (320-340 $\mathrm{kDa})$ and crystallinity degrees (56-60\%). The investigation of regime influence on the process of production of fibers out of PHBV showed that the extrusion stability and extrudate quality were mostly affected by the temperature at the end of the extruder and in the nozzle. We found optimal values of thewse parameters which allowed to calculate maximum stability of extrusion/reception and produce best quality fibers (Table 1). Deviation from the indicated temperatures by several degrees leads to substantial changes in the melt viscosity. For instance, if the temperature is increased by $3-5^{\circ} \mathrm{C}$, the melt viscosity becomes so low that the polymer flow can't bear its own weight. Decrease of the temperature leads to larger viscosity and substantial increase of extruder pressure. The

Table 1. Optimal temperature modes for PHBV copolymers spinning

\begin{tabular}{|c|c|c|}
\hline Hydroxyvalerate inclusions & Temperature mode*, ${ }^{\circ} \mathrm{C}$ & Melting temperature, ${ }^{\circ} \mathrm{C}$ \\
\hline PHB10V $(10 \mathrm{~mol} \%)$ & $155-158-160-162-165$ & 160 \\
\hline PHB15V $(15 \mathrm{~mol} \%)$ & $150-155-157-160-162$ & 158 \\
\hline PHB21V $(21 \mathrm{~mol} \%)$ & $142-144-148-152-155$ & 150 \\
\hline
\end{tabular}

* - distribution of temperatures along the extruder from the feed zone to the nozzle. 
increase of pressure is obviously connected with the fact that the optimal temperatures in the nozzle are very close to the melting temperature and thus, when the temperature is reduced the polymer doesn't have enough time to melt and blocks the nozzle. This causes a sharp pressure jump. The temperatures at the receiving zones of the extruder have a smaller effect on polymer viscosity and can be increased by $5-7^{\circ} \mathrm{C}$, but this can cause a more rapid thermodegradation of the polymer.

Pressure at the nozzle can also be used as a parameter of optimization of fiber production process. In the described experiments the optimal pressure at the nozzle was 35-40 atm. This parameter depends on the type/size of extruder and nozzle, feeding speed, molecular weight of polymer and some other factors. It was established that if the temperature of the receiving pit is 18$20^{\circ} \mathrm{C}$ and the feeding speed is over $25 \mathrm{~m} / \mathrm{min}$, its overall length should be not less than $5 \mathrm{~m}$. This prevents fibers from adhesion at the take-up drum. The length of the receiving pit can be substantially reduced once the temperature of the liquid reaches $60-80^{\circ} \mathrm{C}$. It was stated that the reception speed can vary within wide ranges, from 2-3 to $40 \mathrm{~m} /$ min, without having a considerable effect on the process stability and fiber quality. On the other hand, the diameter of the formed fibers and the degree of molecular orientation in them is directly related to the reception speed. High reception speed (over $30 \mathrm{~m} / \mathrm{min}$ ) led to adhesion of fibers regardless of the temperature of the receiving pit. This fact allows to suppose that adhesion is connected with incomplete crystallization in the newly formed material. To establish a quantitative ratio between reception speed, temperature of receovong pit and thickness of fibers, on the one hand, and crystallization rate, on the other hand, special investigations should be conducted.

The produced fibers were studied with a view to extentability. It was shown that the newly formed extrudates can be extended reaching rather high extension range even at room temperature. However, extension should be made immediately or within 5-7 minutes after extrusion.

To answer the question in what way the storage time of extruded fibers affects their extentability, we conducted special research. Copolymers of each type (with inclusions of hydroxyvalerate 10,15 and $21 \mathrm{~mol} \% \mathrm{t}$ ) were used to form 3-4 samples at various reception speeds, the speed and temperature regime of extrusion being kept at permanent level. Optimal temperature regimes were set for each copolymer (Table 1). The extentability of the samples at room temperature (cold drawing) and on a hot plate was estimated as a function of storage time at room temperature. Measurements were made 1 week, 1 month, and 3 months after the extrusion.

It was stated that the thickness, and consequently the orientation of the extruded fibers is a key factor that determines the extentability. Only fibers with diameter less than $0.3 \mathrm{~mm}$ athat were stored for 1 week appeared to be cold drawable. Table 2 shows, as an example, the characteristics of PHBV fibers (with inclusions of hydroxyvalerate 15 mole percent), produced at various reception speeds and extended at room temperature after 1 month storage. According to the data presented, only PHB15V-1 sample changed its form and necked in, revealing a considerable elongation at break (to $274 \mathrm{MPa}$ ). All other samples demonstrated, at room temperature, typical behaviour for brittle materials.

We investigated the effect of temperature on the deformability of extruded fibers in the range of $40-120^{\circ} \mathrm{C}$. To reach the maximal extension range, visual quality and mechanical properties of fibers, the optimal temperature was set between 80 and $100^{\circ} \mathrm{C}$. At $40^{\circ} \mathrm{C}$ deformability of all fibers hardly differed from extension at room temperature. Extension at 60 and $120^{\circ} \mathrm{C}$ was characterized by instability. It appeared that maximal extension 
Table 2. Mechanical properties of fibers produced at various accepting speeds out of PHBV with 15 mol $\%$ of hydroxyvalerate

\begin{tabular}{|c|c|c|c|c|}
\hline Sample code & $\begin{array}{c}\text { Acception speed, } \\
\mathrm{m} / \mathrm{min}\end{array}$ & $\begin{array}{c}\text { Fiber diameter, } \\
\mathrm{mm}\end{array}$ & Strength, MPa & $\begin{array}{c}\text { Elongation at break, } \\
\%\end{array}$ \\
\hline PHB15V-1 & 40 & 0.30 & 59.8 & 274.0 \\
\hline PHB15V-2 & 35 & 0.35 & 52.3 & 4.0 \\
\hline PHB15V-3 & 25 & 0.45 & 48.6 & 4.5 \\
\hline PHB15V-4 & 10 & 0.80 & 34.3 & 3.1 \\
\hline
\end{tabular}

Table 3. Mechanical properties of fibers made out of various types of PHBV extended up to the maximal extension range

\begin{tabular}{|c|c|c|c|c|}
\hline $\begin{array}{c}\text { Hydroxyvalerate } \\
\text { inclusions }\end{array}$ & $\begin{array}{c}\text { Fiber diameter, } \\
\mathrm{mm}\end{array}$ & Strength, MPa & $\begin{array}{c}\text { Elasticity modulus, } \\
\mathrm{MPa}\end{array}$ & $\begin{array}{c}\text { Elongation at break, } \\
\%\end{array}$ \\
\hline $\begin{array}{c}\text { PHB10V } \\
(10 \mathrm{~mol} \%)\end{array}$ & 0.18 & 306 & 3000 & 24.1 \\
\hline $\begin{array}{c}\text { PHB15V } \\
(15 \mathrm{~mol} \%)\end{array}$ & 0.20 & 281 & 2200 & 32.4 \\
\hline $\begin{array}{c}\text { PHB21 V } \\
(21 \mathrm{~mol} \%)\end{array}$ & 0.17 & 254 & 2100 & 37.9 \\
\hline
\end{tabular}

range at these temperatures depends on the thickness of fibers and parameters of extension (speed and applied load). The reasons for this instability are obviously different at different temperatures. Besides, a considerable number of fibrillation (disintegration) cases was observed at extension temperature of $60^{\circ} \mathrm{C}$ and lower. Thus, within the temperature range $80-100^{\circ} \mathrm{C}$ maximal extension range (7-8) and high mechanical properties (strength $300 \mathrm{MPa}$, elasticity modulus $3 \mathrm{GPa})$ were attained. It should be mentioned that fibers with a larger diameter (up to $0.5 \mathrm{~mm}$ ), can be extended at these temperatures even after 3 month storage. Table 3 shows maximal mechanical characteristics of fibers attained for each type of the investigated PHBV copolymers.

As the mechanical behaviour of fibers produced out of PHBV with different inclusions of hydroxyvalerate appeared to be similar in a qualitative sense, more detailed investigations of the mechanical properties of fibers were made on PHBV samples with inclusions of 15 mole percent of hydroxyvalerate that were extended at $100^{\circ} \mathrm{C}$ (PHB15V_100).
The analysis of extension curves of oriented fibers produced from extrudates with various diameters $(0.30,0.35$ and $0.45 \mathrm{~mm}$ ) (figure) showed that the qualitative nature of deformation doesn't depend on the thickness and, thus, structure of fibers. At the same time the samples had sufficiently large quantitative differences. It is possible to mark out three deformation zones on the curves for all three samples, which makes it similar to the extension curves of polymer elastomers. According to the figure, the zone of linear elasticity showing no visible irreversible phenomena stretches till the first inflection of the extension curve. The values of deformation and stress limiting this zone are practically equal for all investigated fiber samples. In the deformation zone, above the linear elasticity and up to the second inflection, the curve shows visible irreversible deformations that are easy to measure after unloading of samples. Finally, deformations above the second inflection are plastic, i.e. totally irreversible. It should be noted that thin fibers reveal totally irreversible deformations at greater stresses. 


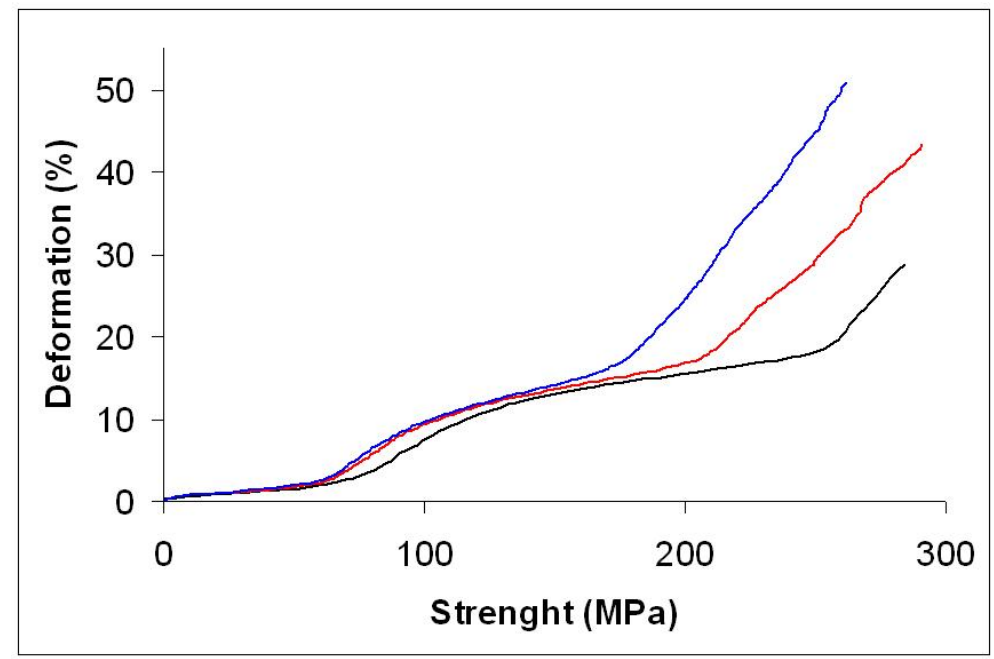

Fig. Extension curves of oriented fibers produced out of PHBV copolymer with $15 \mathrm{~mol} \%$ of hydroxyvalerate (PHB15V) obtained from extrudates of various diameters: $1-0.30 ; 2-0.35 ; 3-0.45 \mathrm{~mm}$

The most important performance parameter of polymer materials is static creep. Measurement of creep is made by measurement of deformation in the material exposed to continuous load during a long-term period. We measured creep deformation of fibers produced from the copolymer with inclusion of 15 mole percent of hydroxyvalerate and extended at $100^{\circ} \mathrm{C}$ (PHB15V_100) during 1 week at loads of 50, 100 and $200 \mathrm{MPa}$ (Table 4). As is seen from the data presented, all three samples demonstrated high resistance to creep deformation in the zone of linear elasticity. However, if the loads exceeded the limit of linear elasticity, resistance of the samples visibly decreased. The resistance was inversely proportional to the thickness of fibers, though the limiting characteristics of all three fibers differ insignificantly. Obviously, a more stable structure forms in thin fibers due to higher orientation, which manifests itself in the location of the plastic deformation zone, in particular. In general, the behaviour of all thress samples at static load is typical for other thermoplastics with glass-transition temperature about or below $0^{\circ} \mathrm{C}$, for example, polypropylene (PP) and polyethylene (PE).

A certain difference was revealed between the fibers out of PHBV and PP and PE during the investigation of reversible deformations under cyclic loads. In this case the samples were loaded at a permanent speed up to the same values as in the creep experiments and then were immediately unloaded. The cycle was repeated 1000 times or till the sample broke (Table 5). In the process of investigation we measured the deformation of samples at maximal load and deformation set after unloading. Like under static load, the samples of PHBV fibers showed high mechanical resistance to cyclic load in the zone of linear elasticity. As expected, deformations in this zone are practically totally reversible even after 1000 loading cycles. Under loading up to $100 \mathrm{MPa}$, the level of deformation reversibility appeared to be rather high, which is, as a rule, more typical for elastomers than for thermoplastics. It was also demonstrated that the mechanical properties of oriented fibers produced out of PHBV after 3 months storage are practically identical to the ones measured immediately after extension. 
Table 4. Static creep of fibers produced out of PHB/PHV with $15 \mathrm{~mol} \%$ of hydroxyvalerate under various loads during 1 week

\begin{tabular}{|c|c|c|c|}
\hline \multirow{2}{*}{ Sample code } & \multicolumn{2}{|c|}{ Deformation (\%) 7 days later under load (MPa): } \\
\cline { 2 - 4 } & 50 & 100 & 200 \\
\hline PHB15V1_100 & 3.0 & 9.8 & 19.7 \\
\hline PHB15V2_100 & 3.1 & 13.5 & 24.8 \\
\hline PHB15V3_100 & 3.1 & 14.2 & 35.1 \\
\hline
\end{tabular}

Table 5. Complete and reversible deformation in fibers produced out of PHBV with $15 \mathrm{~mol} \%$ of hydroxybutyrate subjected to cyclic load

\begin{tabular}{|c|c|c|c|}
\hline \multirow{2}{*}{ Sample code } & \multicolumn{3}{|c|}{$\begin{array}{c}\text { Complete deformation/deformation set (\%) } \\
\text { after 1000 load cycles reaching the load of (MPa): }\end{array}$} \\
\cline { 2 - 4 } & 50 & 100 & 200 \\
\hline PHB15V1_100 & $2.8 / 0.4$ & $7.8 / 3.5$ & $16.7 / 12.8$ \\
\hline PHB15V2_100 & $3.0 / 0.6$ & $10.4 / 4.6$ & $*$ \\
\hline PHB15V3_100 & $3.0 / 0.6$ & $12.3 / 5.0$ & $* *$ \\
\hline
\end{tabular}

* - sample destroyed after 620 cycles

** - sample destroyed after 470 cycles

\section{Discussion}

One of the crucial properties of thermoplastic is the rheological characteristics of melts, first of all, extensional viscosity, "elongation thickness" and increase of viscosity at elongation. These characteristics are important for stabilization of polymers during processing, inclusing such processes as melt spinning (film blowing, extension and doubling of fibers, hot-melt coating etc.). It was stated that the rheological characteristics of PHBV copolymer melts depend to a large extent on processing parameters (Asrar and Pierre, 2000). For instance, shear thinning leads to the increase of termination rate, and the increase of polymer molecular weight results in the increase of shear viscosity and extensional viscosity. It was stated that the influence of molecular weight on extensional viscosity is more considerable than on shear viscosity (Asrar, D’Haene, 2001).

PHA melts are often used for production of fibers, but in the process of melt spinning it's necessary to control crystallization during the extension stage. PHA don't crystallize in order and contain amorphous phase, and fibers can't be extended from amorphous phase melts. For a correct PHA crystallization procedure it's necessary to strictly control the time and temperature of the process for subsequent orientation of fibers aimed at increasing of their strength and necessary physical and mechanical properties. The success of fiber orientation depends on the polymer crystallization kinetics.

There exists a number of works dedicated to the optimization of fiber production out of PHA melts. Theoretically, monofilaments produced by melt spinning can have satisfactory physical and mechanical properties, in particular: breaking strength - over $300 \mathrm{MPa}$, elasticity modulus - up to $10 \mathrm{GPa}$, possessing at the same time a high degree of reversible deformation (More and Sauders, 1998). Japanese scientists in a series of works consider the process of production of monofilaments out of PHB melt and PHBV copolymers, investigating the structure and properties of such fibers (Yamada and Kan, 1995; Yamamoto et al., 1997). PHB crystallized into an orthrhombic vesicular structure with cell parameters $\left(\mathrm{P} 2{ }_{1} 2_{1} 2_{1}\right.$ : $\mathrm{a}=0,576, \mathrm{~b}=1,320$ and 
$\mathrm{c}=0,596 \mathrm{~mm}, \alpha$-form with chains of levorotatory orientation 2/1. X-Ray was used to state that PHB in fibers is represented by two modifications of crystals and has bimodal chain orientation. The chain axes of the first generation crystals are oriented mostly along the fiber axis, while the chain axes of the second generation crystals are oriented crosswise. In the process of subsequent hardening at $100^{\circ} \mathrm{C}$ the percent of populations of the first type crystals considerably prevails over the second population, but the increase of temperature leads to predominance of the second population. It turned out that due to hardening of fibers the percent of crosswise-oriented chains grows, which improves the quality of fibers, increasing their strength. In one of the works the authors (Yamane et al.,2001) studied the structure and mechanical properties of fibers produced by PHB melt spinning using a laboratory singlescrew extruder with $1 \mathrm{~mm}$ diameter round nozzle (the temperature of polymer extrudate was $180^{\circ}$ $\mathrm{C}$, and in the nozzle zone $-170^{\circ} \mathrm{C}$ ). Hardening and orientation of fibers was made six times at different temperatures. The fibers extended at $75,125,150^{\circ} \mathrm{C}$ had strength value of $0,50,100$ $\mathrm{MPa}$, correspondingly. The fibers being extended stayed at the heating zone not more than 2.5 min. There is data showing that the increase of hydroxyvalerate content in the PHBV copolymer, reducing crystallinity and material crystallization time, contributes to a more slow and successful orientation (Katsuhiko, 1994). These results match the results presented in our work that showed the possibilities of successful orientation of fibers made out of PHBV melt.

Japanese researchers under supervision of Professor Doi managed to substantially increase the mechanical stength of PHB monofilaments using a new original "cold-drawn" technology (Iwata et al., 2002). High-polymeric substance (600,000 Da) synthesized by natural and recombinant producer strains was used for production of filaments.
Fibers were spun out of polymer melt into the tank with water having ice temperature. After that the fibers were oriented two times: first, at $0^{\circ}$ $\mathrm{C}$, second - at room temperature. The fibers had high values of strength characteristics: absolute strength $1.4 \mathrm{GPa}$; elasticity modulus $18.1 \mathrm{GPa}$. Improving of this technology and application of the method of fiber production by melt spinning allowed to obtain high-oriented fibers out of polyhydroxybutyrate (Furuhashi et al., 2004). The fibers has the following characteristics: absolute strength - up to $300 \mathrm{MPa}$, elasticity modulus 2,6 MPa, elongation at break $42 \%$. The authors studied the dependence of fiber strength characteristics on the process parameters (spinning temperature, the temperature and time of orientation). According to the data given in the present work, it is possible to produce good quality monofilaments out of PHBV melt taking into account supervision of the process of polymer crystallization. The extentability and deformability of PHBV fibers were thoroughly studied at various temperatures, and as a result, the optimal conditions were found allowing to produce $0.17-0.20 \mathrm{~mm}$ diameter fibers with high mechanical characteristics (strength $306 \mathrm{MPa}$, elasticity modulus $3 \mathrm{GPa}$, elongation at break 24\%) that were stable during the 3 month period of observation. We were the first to obtain and analyze extension curves on which three zones are fixed: linear elasticity, visible deformation and totally irreversible deformation, which is typical for extension curves of polymer elastomers, and showed that the produced fibers possess high mechanical stability under static and cyclic load (up to $100 \mathrm{MPa}$ ); in the linear elasticity zone the deformation of fibers is practically totally irreversible after 1000 load cycles.

\section{Conclusion}

Hydroxybutyrate and hydroxyvalerate copolymers with various inclusions of hydroxyvalerate $(10,15$ and $21 \mathrm{~mol} \%)$ were 
melt-spun and extended to produce oriented monofilaments $0.17-0.20 \mathrm{~mm}$ in diameter with high mechanical characteristics (strength 306 $\mathrm{MPa}$, elasticity modulus $3 \mathrm{GPa}$, elongation at break $24 \%$ ) that were stable during the 3 month period of observation. It was revealed that temperature has a considerable effect on the stability of extrusion and quality of fibers, and the optimal parameter values were revealed (polymer melting temperature and temperature distribution along the extruder from the feed zone to the nozzle). It was stated that the diameter of fibers and degree of orientation ddepend on spinning rate, but changing of the spinning rate within a wide range (from 2-3 to $40 \mathrm{~m} / \mathrm{min}$ ) doesn't affect fiber quality. Fibers produced from PHBV are characterized by high mechanical stability under static and cyclic load (up to $100 \mathrm{MPa}$ ); in the zone of linear elasticity, fiber deformation is practically totally reversible after 1000 load cycles, which is typical of elastomers.

\section{Acknowledgements}

This study was financially supported by the Russian Ministry of Education and the U.S. Civilian Research \& Development Foundation CRDF (Grant № P1MO002); the Program of the RAS Presidium "Fundamental Research to Medicine" (Project № 12.5); the grant of Russian Foundation for Basic Research (RFBR) grant № 07-03-00112-a.

\section{References}

Amass W., Amass A., Tighe B. (1998) A Review of Biodegradale Polymers: Uses, Current Developments in the Synthesis and Characterization of Biodegradable Polyesters, Blends of Biodegradable Polymers and Recent Advances in Biodegradation Studies. Polymer Int. 47: 89-144

Asrar J., Pierre J. (2000) Poly(hydroxyalkanoates) compositions and method of their use in the production of films. European Patent № 0996670A1

Asrar J., D’Haene P. (2001) Polymer blends containing polyhydroxyalkanoates and compositions with good retention of elongation. US Patent № 6191203

Furuhashi Y., Imamura Y., Jikihara Y. (2004) Higher order structures and mechanical properties of bacterial homo poly(3-hydroxybutyrate) fibers prepared by cool-drawing annealing processes. Polymer.45: 5703-5712

Gordeev S.A., Nekrasov Y.P. (1999) Processing and mechanical properties of oriented poly( $\beta$ hydroxybutyrate) fibers J. Mater. Sci. Lett. 18:1691-1692

Gordeev S.A., Nekrasov Y.P., Shilton S.J. (2001) Processing of Gel-Spun Poly( $\beta$-hydroxybutyrate) fibers. J. Appl. Polym. Sci.81:2260-2264

Ishikawa K. (1996) Flexible trembler for use as a medical bag. US Patent № 5480,394

Iwata T., Aoyagi Y., Yamane H. (2002). Mechanical properties, high ordered structure and biodegradability of $\mathrm{P}(3 \mathrm{HB})$ films, fibers and lamellar crystals. Abstract of Int. Symp. Biol.Polym. ISBP-2002)Germany. Münster. 22-26 Sept. P. 48.

Katsuhiko T. (1994) Biopolymer properties and processing // In: Biodegradable and Polymer (Y.Doi and K.Fukuda. Eds.). Amsterdam: Elsevier. P. 362-387.

More G.S., Sauders S.M. (1998) Advances in Biodegradable Polymer. UK, Kapra. Shiropshire. $1000 \mathrm{p}$. 
Trademark "BIOPLASTOTANTM”(2006) Registration certificate № 315652, application № $2006703271 / 50$

Volova T.G. (2004) Microbial polyhydroxyalkanoates - plastic materials of the $21^{\text {st }}$ century (biosynthesis, properties, applications). Nova Science Pub. Inc. NY, USA. 283 p.

Volova T.G., Kalacheva G.S., Steinbüchel A. (2007). Biosynthesis of Multi-component Polyhydroxyalkanoates by the bacterium Wautersia eutropha. J. Siberian Federal University. Biology. 1:91-101.

Williams S.F., Peoples O.P.(1997) Making plastics green: PHA are the new generation of environmentally friendly plastics, easy to make with a little help from genetically engineered bacteria and plants.Chemistry in Britain.32: 29-32

Yamada K., Kan Y. (1995) Murase S. Biodegradable composite fibers with Poly(hydroxyalkanoic acid) core and Poly(alkylene succinate) sheath. Japanese Patent № 07324227.

Yamamoto T., Kimizu M., Kikutani T. (1997) The effect of drawing and annealing conditions on the structure and properties of bacterial Poly(3-hydroxybutyrate-co-hydroxyvalerate) fibers. Int. Polym. Process. 12:29-37.

Yamane H., Terao K., Hiki S., Kawahara Y., Kimura Y., Saito T. (2001a) Processing melt spun Polyhydroxybutyrate Fibers. Polymer.42: 3241-3249. 